\title{
Gödel's Universe and Induced-Matter Theory
}

\author{
J. B. Fonseca-Neto ${ }^{a}$, C. Romero ${ }^{a}$, and F. Dahia ${ }^{b}$ \\ ${ }^{a}$ Departamento de Física, Universidade Federal da Paraíba, \\ C. Postal 5008, 58051-970 João Pessoa, Pb, Brazil and \\ ${ }^{b}$ Departamento de Física, Universidade Federal de Campina Grande, 58109-970, Campina Grande, Pb, Brazil
}

(Received on 15 October, 2005)

\begin{abstract}
We discuss Gödel's universe in the context of the induced-matter theory. We show that the problem of generating Gödel's metric from an extra dimension is equivalent to finding an embedding of Gödel's universe in a Ricci-flat five-dimensional space. On the other hand, according to the Campbell-Magaard theorem, any spacetime can be locally embedded into a five-dimensional pseudo-Riemannian Ricci-flat manifold. We obtain explicitly a global embedding of Gödel's universe which is Ricci-flat and has a non-Lorentzian signature of type $(++---)$.
\end{abstract}

\section{INTRODUCTION}

Einstein's first contact with the idea that our ordinary four-dimensional spacetime might be embedded in a higherdimensional manifold was made through the work of $\mathrm{T}$. Kaluza, sent to him in 1919. From the old Kaluza-Klein[1] model to modern theories of supergravity and superstrings [2] the assumption that extra dimensions may exist, though not observed yet, appears to be closely connected with the belief that all forces of nature are ultimately different aspects of a single entity. Besides the quest for unification there is another motivation for constructing higher-dimensional theories which goes back to Einstein and consists in regarding the physical world as a manifestation of pure geometry[3]. Of these two schemes the latter includes the so-called inducedmatter theory (IMT) or non-compactified Kaluza-Klein theory of gravity, an approach which regards macroscopic matter as being geometrically "induced" by a mechanism that locally embeds our four-dimensional (4D) spacetime in a Ricci-flat five-dimensional manifold [4]. Moreover, it is asserted that only one extra dimension should be sufficient to explain all the phenomenological properties of matter. One interesting point is that the matter "generated" by this process is of a very general kind, i.e. any energy-momentum tensor can be produced by choosing the appropriate embedding, a result which is mathematically supported by a powerful theorem of differential geometry due to Campbell and Magaard [5, 6].

In 1949, Kurt Gödel [7] found a solution of Einstein's field equations which soon became very popular because it described a spacetime possessing very strange properties. For example, the model admitted the existence of timelike closed curves, thereby violating global causality. Although not viable as a physical model of our universe, Gödel's solution has some historical importance as it certainly stimulated a great deal of research on questions of causality and global properties of relativistic spacetimes $[8,9]$.

Due to its peculiarity, different aspects of the so-called Gödel's universe have always been studied with interest. For example, Rosen [10] in 1965, was able to characterize Gödel's model as a four-dimensional hypersurface embedded in a pseudo-Euclidean space with ten dimensions. A recent motivation to study the embedding of Gödel's solution comes from the induced-matter proposal. It is known that Wesson and co- laborators have shown how to obtain from five-dimensional vacuum (or Ricci-flat) spaces a number of known solutions of the Einstein equations (regarded as hypersurfaces in five dimensions) whose energy-momentum tensor is generated by the extra-dimension [4]. In fact, the energy-momentum thus generated corresponds to the extrinsic curvature of the fourmanifold embedded in five-dimensional vacuum space [11]. It has been later realised [12] that any energy-momentum tensor can be generated in this way, provided that any solution of Einstein's equations has an embedding into a five-dimensional Ricci-flat solution, and this is almost precisely the content of the Campbell-Magaard theorem [5, 6]. Therefore, according to the this theorem, it is possible, to locally embed Gödel's solution in a five-dimensional Ricci-flat pseudo-Riemannian space. From the standpoint of the induced-matter theory, that means it must be possible to geometrically generate a source of matter and energy which is the source of Gödel's universe with all its peculiarities.

To be more precise let us recall the content of the CampbellMagaard theorem $[5,6]$. It states that any $n$-dimensional pseudo-Riemannian manifold $\left(M^{n}, g\right)$ can be locally, analytically and isometrically embedded in a Ricci-flat $(n+1)$ dimensional manifold $\left(N^{n+1}, \widetilde{g}\right)$. Since its "rediscovery" in the nineties [12] the theorem has found a number of applications and has been discussed in various contexts in the literature [13-24]. Therefore, in view of the Campbell-Magaard theorem one would like to look at Gödel's solution as a hypersurface embedded in a five-dimensional Ricci-flat space.

\section{THE EMBEDDING OF GÖDEL'S UNIVERSE IN FIVE DIMENSIONS}

Gödel's metric may be expressed in the form

$d s^{2}=d t^{2}-d x^{2}+\frac{1}{2} \exp (2 \sqrt{2} w x) d y^{2}-d z^{2}+2 \exp (\sqrt{2} w x) d t d y$

where $w$ is a constant.

In this section we shall show how to obtain the embedding of Gödel's spacetime in a five-dimensional Ricci-flat space, the metric of which has signature $(++---)$. When $n \geq 3$, the Campbell-Magaard alows us to lower the number of dimensions of the embedding space $N^{n+1}$ from $n(n+1) / 2$ to 
$n+1$, as long as $N^{n+1}$ be Ricci-flat. However, we shall not employ directly the Campbell-Magaard; instead we shall make use of the following theorem due to Magaard [6]:

Theorem (Magaard). Let $\left(M^{n}, g\right)$ be a $n$-dimensional pseudo-Riemannian manifold, $\left\{x^{\mu}\right\}$ a local coordinate system of a neighbourhood $U$ of $p \in M^{n}$, with coordinates $\left(x_{p}^{1}, \ldots, x_{p}^{n}\right)$ defined by the parametrization $\mathbf{x}: U \rightarrow M^{n}$. A sufficient and necessary condition for $\left(M^{n}, g\right)$, with line element $d s^{2}=g_{\alpha \beta}(x) d x^{\alpha} d x^{\beta}$, to be locally, isometrically and analytically embedded in a $(n+1)$-dimensional manifold $\left(M^{n+1}, \widetilde{g}\right)$ is that there exist analytical functions

$$
\begin{gathered}
\bar{g}_{\alpha \beta}=\bar{g}_{\alpha \beta}\left(x^{1}, \ldots, x^{n}, x^{n+1}\right) \\
\bar{\phi}=\bar{\phi}\left(x^{1}, \ldots, x^{n}, x^{n+1}\right)
\end{gathered}
$$

definided in an open set $D \subset \mathbf{x}(U) \times \mathbf{R}^{n}$ containing the point $\left(x_{p}^{1}, \ldots, x_{p}^{n}, 0\right)$, satisfying the following conditions:

$$
\bar{g}_{\alpha \beta}\left(x^{1}, \ldots, x^{n}, 0\right)=g_{\alpha \beta}\left(x^{1}, \ldots, x^{n}\right)
$$

in an open set of $\mathbf{x}(U) ; \bar{g}_{\alpha \beta}=\bar{g}_{\beta \alpha},\left|\bar{g}_{\alpha \beta}\right| \neq 0 ; \bar{\phi} \neq 0$, and that

$$
d \bar{s}^{2}=\bar{g}_{\alpha \beta} d x^{\alpha} d x^{\beta}+\varepsilon \bar{\phi}^{2} d x^{n+1} d x^{n+1}
$$

with $\varepsilon^{2}=1$, represents the line element of $M^{n+1}$ in a coordinate neighbourhood $V$ of $M^{n+1}[6,26]$.

In the light of the above theorem let us take $n=4, \varepsilon=1$, $\phi=-k^{2}$, where $k$ is a constant, and the set of analytical functions $\left\{\bar{g}_{\alpha \beta}(t, x, y, z, \psi\}\right.$ [36], $(\alpha, \beta=0,1,2,3)$ the nonnull elements of which are $\bar{g}_{00}=1 ; \bar{g}_{02}=\bar{g}_{20}=\exp (\sqrt{2} w(x+$ $k \psi)) ; \bar{g}_{11}=-1 ; \bar{g}_{22}=\frac{1}{2} \exp (2 \sqrt{2} w(x+k \psi)) ; \bar{g}_{13}=\bar{g}_{31} ; \bar{g}_{33}=$ -1 . Clearly the conditions $\bar{g}_{\alpha \beta}=\bar{g}_{\beta \alpha}, \bar{\phi} \neq 0$ are satisfied, and also $\left|\bar{g}_{\alpha \beta}\right|=-\frac{1}{2} \exp (2 \sqrt{2} w(x+k \psi)) \neq 0$. Moreover, $\bar{g}_{\alpha \beta}(t, x, y, z, 0)=g_{\alpha \beta}(t, x, y, z)$, hence the functions $g_{\alpha \beta}$ may be identified with the components of Gödel's metric written in the form (1). We conclude, therefore, from the above theorem that the Gödel's universe can be embedded in a fivedimensional space $M^{5}$ with metric given by

$$
\begin{aligned}
d S^{2} & =d t^{2}-d x^{2}+\frac{1}{2} \exp (2 \sqrt{2} w(x+k \psi)) d y^{2}-d z^{2} \\
& +2 \exp (\sqrt{2} w(x+k \psi)) d t d y+k^{2} d \psi^{2}
\end{aligned}
$$

the embedding taking place for $\psi=0$, i.e. by choosing the embedding functions given by $t \rightarrow t, x \rightarrow x, y \rightarrow y, z \rightarrow z, \psi=$ 0 .

If we calculate [37] the components ${ }^{(5)} R_{a b}$ of the Ricci tensor directly from (5) we get ${ }^{(5)} R_{a b}=0$. We see then that the five-dimensional manifold $M^{5}$, in which Gödel's universe appears as the hypersurface $\psi=0$, is a Ricci-flat space, and that proves our claim.
Let us conclude this section with the following comment. The manifold on which Gödel's metric is defined is $R^{4}$, i.e. $-\infty<t, x, y, z<\infty$ [25], and it is clear that the present embedding takes the whole of $R^{4}$ into $M^{5}$, irrespective of the domain chosen for $\psi$. Moreover, we see that the embedding functions and the metric of the embedded spacetime are analytic in $R^{4}$ while the metric of embedding space is analytical in $M^{5}$. It turns out then that in spite of the local character of the theorem mentioned previously in this particular case the embedding found happens to be global ( a global version of the CampbellMagaard theorem has been discussed recently in [27] ). It is interesting to have a look at the components of the extrinsic curvature tensor $\Omega_{\alpha \beta}$ of the hypersurface $\psi=$ const of $M^{5}$. In the coordinates of (5) it can easily be shown that $\Omega_{\alpha \beta}$ is given by $\Omega_{\alpha \beta}=-\frac{1}{2 k} \frac{\partial \bar{g}_{\alpha \beta}}{\partial \psi}$ [18], so that the nonvanishing components of $\Omega_{\alpha \beta}$ are $\Omega_{02}=\Omega_{20}=-\frac{1}{2} \sqrt{2} w \exp (\sqrt{2} w(x+k \psi))$, $\Omega_{22}=-\sqrt{2} w \exp (2 \sqrt{2} w(x+k \psi))$.As we see, the extrinsic curvature is also well-behaved (analytical) everywhere for any hypersurface of the foliation $\psi=$ const, in particular for $\psi=0$. As a consequence of the global caracter of the embedding, all global properties so characteristics of Gödel's universe, such as the existence of closed timelike curves, are preserved in $M^{5}$.

\section{FINAL REMARKS}

We would like to call attention for the fact that the space $\left(M^{5}, \widetilde{g}\right)$, which is a solution of the Einstein vacuum field equations in five dimensions, has the peculiarity of possessing a non-Lorentzian (ultra-hyperbolic) metric, with two timelike dimensions. Spaces of these kind have been studied recently, mainly in connection with the idea that massless particles in five dimensions may appear "massive" when viewed from four-dimensional spacetime [28-30]. There are also claims that two times theories may find some motivation in M-theory [31]. On the other hand, examples of embedding spaces with extra timelike dimensions are many, and include, for instance, the embedding of the Schwarzschild spacetime in a six-dimensional flat manifold obtained for the first time by Kasner [32, 33]. Isometric embeddings in flat spaces with two times have also been investigated in the context of branes [34]. Finally, it is interesting to note that it is not possible to globally embed a spacetime which is not globally hyperbolic into a pseudo-euclidean space with only one timelike dimension [35]. We do not know whether a similar result holds in the case of Ricci-flat embedding spaces [38].

\section{ACKNOWLEDGEMENT}

The authors would like to thank CNPq-FAPESQ (PRONEX) for financial support. 
[1] T. Kaluza, Sitz. Preuss. Akad. Wiss. 33, 966 (1921). O. Klein, Z. Phys. 37, 895 (1926)

[2] P. Collins, A. Martin and E. Squires, Particle Physics and Cosmology, Ch. 13, Wiley, New York, 1989

[3] A. Einstein, The Meaning of Relativity, p. 129, Princeton University Press, Princeton (1956). J. A. Wheeler, Einstein's Vision, Springer, Berlin (1968). A. Salam, Rev. Mod. Phys. 52 , $525(1968)$

[4] J. M. Overduin and P. S. Wesson, Phys. Reports, 283, 303 (1997). P. S. Wesson, Space-Time-Matter (World Scientific, Singapore, 1999)

[5] J. E. Campbell, A course of Differential Geometry (Clarendon Press, Oxford, 1926)

[6] L. Magaard, Zur Einbettung Riemannscher Räume in EinsteinRäume und konform-euklidische Räume, $\mathrm{PhD}$ thesis, University of Kiel (1966)

[7] K. Gödel, Rev. Mod. Phys. 21, 447 (1949)

[8] E. H. Kronheimer and R. Penrose, Proc. Camb. Phil. Soc. 63, 481 (1967)

[9] R. Geroch, J. Math. Phys. 8, 782 (1967)

[10] J. Rosen, Rev. Mod. Phys. 32, 204 (1965)

[11] M. D. Maia, gr-qc/9512002

[12] C. Romero, R. Tavakol, and R. Zalaletdinov, Gen. Rel. Grav. 28, 365 (1996)

[13] J. E. Lidsey, C. Romero, R. Tavakol, and S. Rippl, Class. Quantum. Grav. 14, 865 (1997)

[14] J. E. Lidsey, Phys. Lett. B 417, 33 (1997)

[15] A. G. Agnese and M. La Camera, Nuovo Cimento Soc. Ital. Fis., B 115, 119 (2000)

[16] E. Anderson and J. E. Lidsey, Class. Quantum Grav. 18, 4831 (2001)

[17] F. Dahia and C. Romero, J. Math. Phys. 43, 3097 (2002)

[18] F. Dahia and C. Romero, J. Math. Phys. 43, 5804 (2002)
[19] S. S. Seahra and P. S. Wesson, Class. Quantum Grav. 20, 1321 (2003)

[20] E. Anderson, F. Dahia, J. Lidsey, and C. Romero, J. Math. Phys. 44, 5108 (2003)

[21] F. Dahia and C. Romero, Class. Quantum Grav. 21, 927 (2004)

[22] S. Chervon, F. Dahia, and C. Romero, Phys. Lett. A 326, 171 (2004)

[23] E. Anderson, gr-qc/0409122

[24] F. Dahia and C. Romero, Class. Quantum Grav. (to appear)

[25] S. W. Hawking and G. F. R. Ellis, The Large Structure of SpaceTime (Cambridge University Press, Cambrige,England, 1973).

[26] F. Dahia, The embedding of spacetime in five dimensions and the generalization of Campbell-Magaard theorem, $\mathrm{PhD}$ thesis, Universidade Federal da Paraíba, Brazil (2001)

[27] N. I. Katzourakis, math.ph/0407067

[28] P. S. Wesson, Mod. Phys. Lett. B 538, 159 (2002)

[29] D. Youm, Mod. Phys. Lett. A 16, 2371 (2001)

[30] D. Youm, Phys. Rev. D 62, 084002 (2000)

[31] S. Vongehr, hep-th/9907034

[32] E. Kasner, Am. J. Math. 43, 130 (1921)

[33] J. Kocinski and M. Wierzbicki, gr-qc/0110075

[34] L. Andrianopoli, M. Derix, G. W. Gibbons, C. Herdeiro, A. Santambrogio, and A. V. Proeyen, Class. Quantum Grav. 17, 1875 (2000)

[35] R. Penrose, Rev. Mod. Phys. D 37, 215 (1965)

[36] We can choose the range of the new coordinates to be given by $-\infty<t, x, y, z, \psi<-\infty$.

[37] This can be done quickly and efficiently by employing algebraic computation programs such as SHEEP or GRTensor.

[38] This article is an abridged and simplified version of our paper "Embedding Gödel's universe in five dimensions" to appear in Modern Physics Letters A 